\title{
Informe editorial, número 6 (2020)
}

\author{
Jaime Escribano Pizarro y Carme Melo Escrihuela
}

\section{GRACIAS POR VUESTRO APOYO}

Tras un año de trabajo, no podemos comenzar de otro modo que mostrando nuestra gratitud a todos los autores y autoras que han elegido TERRA para hacer difusión de sus investigaciones, trabajos y experiencias profesionales en el ámbito del desarrollo local. Gracias a ellos, y al trabajo de todos los miembros del Consejo Editorial y del Consejo Asesor, es posible contar con un número más en nuestra corta existencia.

Como principal objetivo, para una revista todavía sin indexar en los rankings de referencia de la investigación española, garantizar nuestra continuidad sigue siendo un reto mayúsculo. No obstante, por la cantidad de originales recibidos y publicados en este número, tras su correspondiente evaluación externa y aceptación, consideramos que estamos en el camino a seguir. De hecho, incluso podemos plantearnos ya, para este mismo año 2020, incrementar los números publicados de forma anual, y pasar así de uno (el actual) a dos (cuya aparición tendrá lugar a finales de año).

Desde julio de 2019, la regularidad en la recepción de originales se ha hecho constante al lograrse una media de dos textos por mes. Algunos, como artículos o notas de investigación, y otros directamente como reseñas o experiencias y buenas prácticas. Pero no solo la cantidad ha incrementado y su llegada se ha hecho relativamente constante, también la calidad de los textos recibidos es, cada vez, mayor. Las diversas acciones iniciadas hace un año han contribuido a ello, por lo que no podemos más que seguir aplicándolas con renovado esfuerzo: la difusión de TERRA en el ámbito académico e investigador latinoamericano y español, a través de la participación en reuniones y/o jornadas científicas relacionadas con el Desarrollo Local; la aceptación de originales en distintos idiomas (español, catalán, portugués, inglés y francés); la actualización constante de la página web de la revista, como principal medio de difusión e imagen de TERRA, en particular a través de la sección de textos pre-publicados; la invitación a proponer y participar en monográficos derivados de proyectos de investigación; la conexión con juniors investigadores interesados en conseguir dar salida a sus trabajos, en un entorno de calidad contrastada; la divulgación de los contenidos entre colectivos especialmente interesados en el ámbito del Desarrollo Local, tanto desde el mundo académico (a través de distintos masters universitarios) como profesional (vía asociaciones, organismos públicos y/o foros especializados); etc.

\section{REALIZACIONES Y TAREAS PENDIENTES}

Varias han sido las realizaciones efectuadas en este año que, consideramos, han tenido un impacto destacado en el alcance logrado por TERRA y su consolidación como revista de referencia en el ámbito del Desarrollo Local. Por un lado, la participación como entidad 
colaboradora en reuniones y congresos científicos nacionales e internacionales, por otro, la cooperación con cátedras institucionales-universitarias para la difusión de trabajos académicos con clara temática territorial, realizados en el seno de las mismas y, por último, el trabajo realizado por parte del Consejo Editorial en temas de formatos de presentación y procesos de difusión de los textos aceptados para su publicación, con los que dotar a estos de mayor información sobre sus autores y contenidos y, por tanto, posibilidades de empleo por parte de otros investigadores.

En el primer caso, la colaboración y presencia en reuniones científicas se ha materializado en tres ocasiones: i) el XXVI Congreso de la Asociación Española de Geografía (AGE): "Crisis y espacios de oportunidad. Retos para la Geografía", en la Universitat de València, Valencia (España), del 22 al 25 de octubre de 2019; ii) el V Congreso de Desarrollo Local, de la Universidad de Cartagena, en Cartagena de Indias (Colombia), del 29 de octubre al 01 de noviembre de 2019; y iii) el III Simposium de Jóvenes Investigadores en Geografía (SIJIG), en la Universitat de Barcelona, Barcelona (España), del 27 al 29 de mayo de 2020, pero que finalmente se canceló por la situación de Estado de Alarma declarada ante la pandemia generada por el COVID-19 (Figura 1).

Figura 1. Ejemplo de participación en reuniones científicas

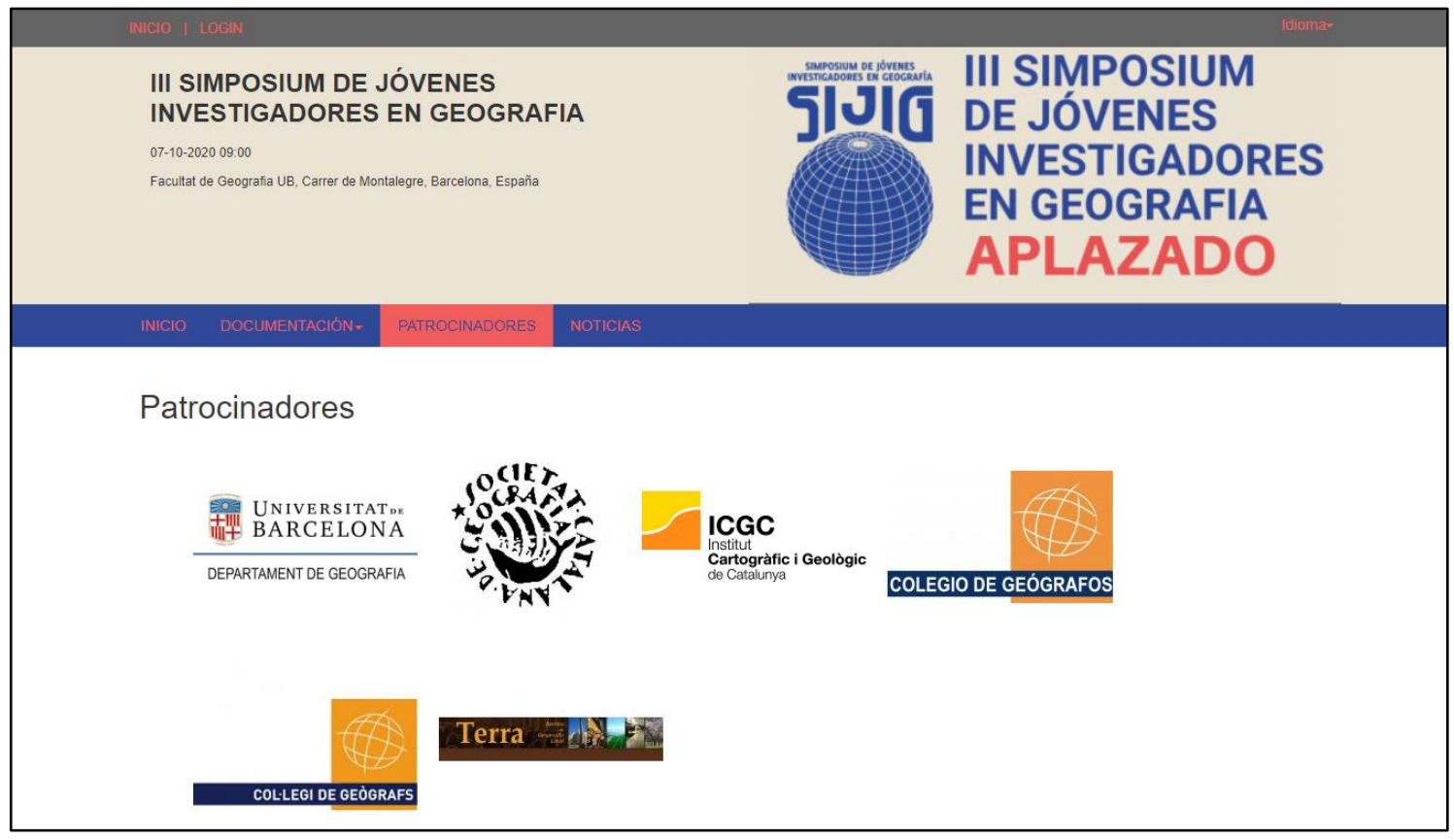

Fuente: http://ub.symposium.events/47609/sponsors/iii-simposium-de-jovenes-investigadores-engeografia.html (consulta realizada el 17/07/2020).

En el segundo caso, la cooperación con cátedras institucionales-universitarias se ha concretado, por el momento, con distintas cátedras de la Universitat de València, de las que ya en el presente número seis se recogen varias aportaciones: i) la Cátedra Ciudad de Cullera; ii) la Cátedra Universitaria Ajuntament d'Ontinyent-Caixa Ontinyent-Eset para la innovación y configuración de territorios inteligentes; iii) la Cátedra Participación Ciudadana y Paisajes Valencianos; iv) la Cátedra Horta de València, Territorio Metropolitano; y v) la Cátedra AVANT (Agenda Valenciana Antidespoblament). 
Y, por último, en el tercer caso, las acciones desarrolladas alrededor de los textos aceptados para su publicación atienden, por un lado, a cuestiones de difusión, por otro, a distintas acciones en cuanto a la edición y la presentación de los originales, y, por último, a una estrategia de internacionalización. En primer lugar, se ha apostado por dar la más rápida difusión posible de todo tipo de texto recibido y aceptado a través de la creación de un apartado de "prepublicaciones" en la web de la revista ${ }^{1}$. En este, aparecen así todos los artículos, notas, experiencias y reseñas desde el mismo momento en que han sido maquetados en su versión definitiva, a expensas únicamente de la paginación final que adquirirá dicho texto en el número de la revista en el que se publique, pero contando con todos los demás datos válidos para poder ser empleados en cualquier proceso de acreditación o concurso público, como el DOI, la identificación y filiación de los autores, etc. De este modo, atendemos a una de las peticiones más habituales de los autores de nuestros originales, y es la inmediata disponibilidad de sus trabajos, una vez aceptados por los evaluadores, para su citación y referenciación por parte de otros investigadores interesados en sus mismas temáticas de desarrollo local. Por consiguiente, los textos aparecen disponibles en unos pocos meses, en lugar de quedar inaccesibles desde su aprobación hasta la publicación on-line del correspondiente número.

En segundo lugar, en cuanto a la edición y la presentación de originales, se ha acordado que los textos que aparezcan como artículos en la revista incorporen, aparte de las secciones habituales (resumen, palabras clave, aportación del autor -organizada habitualmente en una introducción, metodología, resultados y discusiones, conclusiones y referencias), una primera de ideas clave, un resumen extendido en inglés, y una concreción de la contribución que al texto se realiza por parte de cada autor, cuando este es firmado por más de un responsable.

En cuanto a las ideas clave, se propone a los autores que aporten entre tres a cinco frases, de no más de 15 palabras cada una, a modo de idea fundamental del texto. Esta puede ser de cualquier tipo, relacionada con su metodología o con sus resultados, por ejemplo, pero siempre intentando que difiera de la información presentada en el resumen "corto" inicial (el habitualmente solicitado, de no más de 200 palabras). Su presentación se hace, además, en los tres idiomas fundamentales para la revista: español, inglés y catalán. En relación al resumen extendido en inglés, se pretende que los autores, en un máximo de 1.500 palabras (1.000 como mínimo), expongan los contenidos más relevantes de su aportación, siguiendo la organización habitual del texto, es decir, una breve presentaciónjustificación, la explicación metodológica resumida, los resultados más relevantes y las conclusiones básicas. Esta aportación en lengua inglesa, lógicamente solo es demandada en aquellos casos en los que el texto no esté escrito en inglés, siendo, en todo caso, la traducción aportada única y exclusivamente responsabilidad del autor.

Por lo que respecta a la contribución que realizan los autores del texto, se trata de una solicitud destinada a artículos de más de un autor, en la que, mediante una tabla, se recogen diferentes rasgos relacionados con el proceso de diseño, realización y redacciónedición del texto, y el aporte que a cada uno de ellos realizan los distintos autores de la investigación. Este aporte se expresa en porcentaje, siguiendo el listado de ítems propuesto por CRediT (Tabla 1). Si bien la demanda de este tipo de información no es habitual en las revistas de Ciencias Sociales, sí que lo es, por su parte, en el ámbito de las Ciencias Naturales. Aparte, consideramos que contribuye a esclarecer la implicación de los autores en la elaboración de su trabajo, aspecto cada vez más demando en los actuales

\footnotetext{
${ }^{1}$ https://ojs.uv.es/index.php/TERRA/about/editorialPolicies\#custom-3
} 
procesos de acreditación a figuras universitarias, u obtención de reconocimientos por la actividad investigadora realizada.

Tabla 1. Información relativa a la "Contribución de los autores"

\begin{tabular}{|c|l|c|c|c|c|}
\hline \multicolumn{1}{|c|}{ ITEM } & \multicolumn{1}{|c|}{ Autor 1 } & Autor 2 & Autor x+1 & Total \\
\hline 1 & Conceptualización & $-\%$ & $-\%$ & $-\%$ & $100 \%$ \\
\hline 2 & Tratamiento de los datos & $-\%$ & $-\%$ & $-\%$ & $100 \%$ \\
\hline 3 & Análisis formal & $-\%$ & $-\%$ & $-\%$ & $100 \%$ \\
\hline 4 & Acceso financiación & $-\%$ & $-\%$ & $-\%$ & $100 \%$ \\
\hline 5 & Investigación & $-\%$ & $-\%$ & $-\%$ & $100 \%$ \\
\hline 6 & Metodología & $-\%$ & $-\%$ & $-\%$ & $100 \%$ \\
\hline 7 & Gestión del proyecto & $-\%$ & $-\%$ & $-\%$ & $100 \%$ \\
\hline 8 & Recursos & $-\%$ & $-\%$ & $-\%$ & $100 \%$ \\
\hline 9 & Software & $-\%$ & $-\%$ & $-\%$ & $100 \%$ \\
\hline 10 & Supervisión & $-\%$ & $-\%$ & $-\%$ & $100 \%$ \\
\hline 11 & Validación & $-\%$ & $-\%$ & $-\%$ & $100 \%$ \\
\hline 12 & Visualización & $-\%$ & $-\%$ & $-\%$ & $100 \%$ \\
\hline 13 & Redacción (borrador) & $-\%$ & $-\%$ & $-\%$ & $100 \%$ \\
\hline 14 & Redacción final (revisión y edición) & $-\%$ & $-\%$ & $-\%$ & $100 \%$ \\
\hline
\end{tabular}

Para más información, visitar CRediT: https://casrai.org/credit/.

Estos tres aspectos son completamente voluntarios, aunque desde la dirección de la revista se recomienda encarecidamente su realización a los autores de los textos dirigidos a su publicación como artículos. En el caso de los que se dirigen a la sección de Notas y Avances de investigación, las ideas clave y el resumen extendido son omitidos. No obstante, estos dos aspectos son considerados básicos para el tercer punto relacionado con las acciones desarrolladas alrededor de los textos aceptados para su publicación: la internacionalización de nuestra revista. Para poder conseguir un mayor conocimiento de los contenidos que aparecen publicados en TERRA, estos deben ser difundidos en varios idiomas. Y junto al español, clave para la difusión de la misma en España y Latinoamérica, el inglés aparece como la vía más adecuada para llegar a una mayor cantidad de lectores e investigadores interesados en el Desarrollo Local. Además, de este modo seguimos la vía iniciada por otras muchas revistas científicas de Ciencias Sociales, similares a TERRA, empleadas como referentes a partir de las que tomar decisiones para mejorar el posicionamiento y reconocimiento de nuestra revista.

Otro conjunto de realizaciones efectuadas desde el mes de julio de 2019, es la inclusión de TERRA en nuevas bases bibliográficas y la adhesión a sellos de reconocimiento por una investigación de calidad (Tabla 2). E igualmente, la actualización de las Normas de Publicación o Guía/Directrices para Autores, en particular, lo referente a las nuevas indicaciones introducidas por APA en su 7a edición (octubre de 2019) sobre la citación y las referencias bibliográficas, así como la inclusión de los tres aspectos antes comentados relacionados con los formatos de presentación y procesos de difusión de los textos aceptados para su publicación. 
Tabla 2. Presencia de TERRA en bases bibliográficas, plataformas de evaluación y directorios (2015-2020)

\begin{tabular}{|c|c|c|c|}
\hline & $\begin{array}{c}\text { Antes de febrero de } \\
2019\end{array}$ & $\begin{array}{c}\text { De febrero a junio } \\
\text { de 2019 }\end{array}$ & $\begin{array}{c}\text { De julio a junio de } \\
2020\end{array}$ \\
\hline Dialnet & $\mathrm{X}$ & & \\
\hline MIAR & & $\mathrm{X}$ & \\
\hline Google Scholar & & $\mathrm{X}$ & \\
\hline Latindex (Directorio) & & $\mathrm{X}$ & $\mathrm{X}(1)$ \\
\hline Dulcinea & & & $\mathrm{X}$ \\
\hline CIRC & & & $\mathrm{X}$ \\
\hline Redib & & & $\mathrm{X}$ \\
\hline $\begin{array}{c}\text { Portal de Revistas Ibéricas de } \\
\text { Geografía }\end{array}$ & & & $\mathrm{X}$ \\
\hline $\begin{array}{c}\text { DORA (Declaración de San } \\
\text { Francisco) }\end{array}$ & & & $\mathrm{X}$ \\
\hline BASE & & & $\mathrm{X}$ \\
\hline Crossref & & & \\
\hline WorldCat & & & \\
\hline
\end{tabular}

Fuente: elaboración propia a partir de la información disponible en la web de TERRA. (1) Desde octubre de 2019 TERRA está clasificada en la plataforma CIRC con la categoría D.

Gran parte de estas realizaciones han sido posible gracias a los 200,00 euros de apoyo económico otorgados en 2019 por parte de la dirección de la sede de la Universitat de València del IIDL. Recordemos que, con anterioridad, TERRA no disponía de presupuesto alguno, funcionando durante sus cinco primeros años de existencia con nula financiación. Para este año 2020, no solo se ha mantenido la partida en los presupuestos del IIDL-Valencia, sino que incluso se ha incrementado en 300,00 euros más, llegando así a un total de 500,00 euros. Este hecho es, sin duda, un claro ejemplo del respaldo de la dirección del IIDL-Valencia al crecimiento de TERRA, así como muestra más de lo que entendemos, confianza en la labor realizada hasta la fecha. Y, a la vez, conlleva un reto y compromiso mayor por parte de la dirección de la revista, con tal de mantener la dinámica iniciada en cuanto a realizaciones y resultados obtenidos.

En concreto, los 200,00 euros concedidos durante el año 2019 se emplearon en diferentes estrategias de difusión (a través de trípticos y posters) (Figura 2 y 3), destinadas en ambos casos a garantizar la presencia de TERRA en el XXVI Congreso de la Asociación Española de Geografía (AGE), celebrado en la Universitat de València (España), del 22 al 25 de octubre de 2019; y el V Congreso de Desarrollo Local de la Universidad de Cartagena (Cartagena de Indias, Colombia), del 29 de octubre al 01 de noviembre de 2019.

En cuanto a las tareas pendientes y previstas realizar en los próximos meses, tenemos:

a) Traducir la web de la revista al inglés, conforme a la estrategia de internacionalización iniciada mediante otras acciones ya indicadas con anterioridad (ideas clave y resumen extendido en inglés), e igualmente, al idioma cooficial de la Universidad de Valencia y de la Universitat Jaume I, el catalán. En esta misma línea de traducción, se abordará también la referida a las actuales Normas de Publicación o Guía/Directrices para Autores, y la del Formulario para la Evaluación Externa. 
Figura 2. Tríptico confeccionado para la difusión de TERRA durante 2019

\section{Terra}

TERRA. Revista de Desarrollo Local es una revista de periodicidad anual que recoge la reflexión académica y la práctica política que tiene lugar en este campo de estudio y de trabajo.

Dada la propia naturaleza del desarrollo local, entendido en su más amplio sentido, se trata de una plataforma para la difusión de la reflexión teórica más avanzada, pero también de las mejores prácticas que en el campo del desarrollo local se están implementando en el contexto español, europeo e iberoamericano y, de esta manera, sirve como punto de encuentro de académicos, gestores y políticos.

Para lograr este objetivo, TERRA cumple con todos los niveles de exigencia propios de una revista científica basada en la selección de originales para su publicación a partir de un proceso de evaluación por pares ciegos.

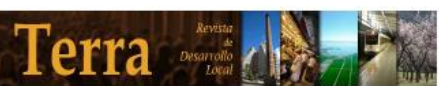

TERRA está presente en:

O Dialnet

latindex

MIAR

C.I.R.C.

8. Google

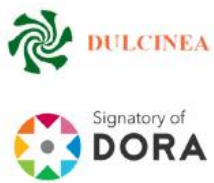

https://ois.uv.es/index.php/TERRA
Terra

TERRA.

Revista de

Desarrollo

Local

Instituto Interuniversitario de

Desarrollo Local (IIDL)

Universidad de Valencia y

Universidad Jaume I (Castellón) España

Revista Open-Access

ISSN: e-2386-9986

journal.terra@gmail.com

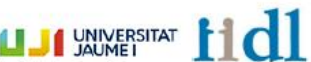

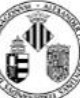

\section{Terra $a$ and}

\section{Terra $=$ and}

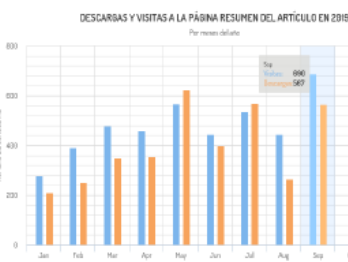

nviado $y$ reg

plataforma, hasta su aceptación definitiva y

prepublicación on-line

Varias políticas de sección...

1. Doble ciego externo:

1.1. Artículos (miscelánea y monográficos)

1.2. Notas y avances de investigación

2. Revisión Consejo Editor:

2.1. Experiencias y Buenas Prácticas 2.2. Reseñas

\section{Terra}

Frecuencia publicación Anual: un número al año (julio) Opción "preprint" on-line con DOI

\begin{tabular}{|c|c|c|c|c|c|}
\hline \multirow{2}{*}{ 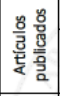 } & \multicolumn{3}{|c|}{ Datos absolutos } & \multicolumn{2}{|c|}{ En porcentaje } \\
\hline & \begin{tabular}{|l} 
Ámbito \\
español
\end{tabular} & $\begin{array}{c}\text { Ámbito } \\
\text { latino- } \\
\text { americano }\end{array}$ & Total & $\begin{array}{l}\text { Ámbito } \\
\text { español }\end{array}$ & $\begin{array}{l}\text { Ámbito } \\
\text { latino- } \\
\text { americano }\end{array}$ \\
\hline $\begin{array}{c}\begin{array}{c}\text { No } 1 \\
\text { (2015) }\end{array} \\
\end{array}$ & 2 & 3 & 5 & 40 & 60 \\
\hline $\begin{array}{c}\text { №2 } \\
(2016)\end{array}$ & 4 & 2 & 6 & 67 & 33 \\
\hline $\begin{array}{c}\text { №3 } 3 \\
(2017)\end{array}$ & 4 & 3 & 7 & 57 & 43 \\
\hline $\begin{array}{c}\text { № 4 } \\
(2018)\end{array}$ & 2 & 3 & 5 & 40 & 60 \\
\hline $\begin{array}{l}\text { № } 5 \\
2019) \\
\end{array}$ & 6 & 3 & 9 & 67 & 33 \\
\hline Total & 18 & 14 & 32 & 56 & 44 \\
\hline
\end{tabular}

DIRBCTOR
Jaime Escribano, Geografia. Universitat de Valencia, España Carme Melo, Ciencias Politicas. Universitat de Valencia, España CONSETO EDITOR María Raquel Agost, Psicologia Social Universitat Jaime I, España Complutense de Madrid, Espana and slands, Reino Unido Internacional de Barcelona (UNBBA) España Cretan Remus, Geografía West University of Timisoara, Rumanía Joaquín Martín Ciencias Politicas. Universitat de Valencia, Espana

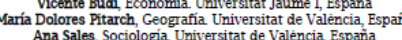

Fuente: elaboración propia. 
Figura 3. Póster confeccionado para la difusión de TERRA (2019) en el V Congreso Internacional de Desarrollo Local (Cartagena de Indias, Colombia)

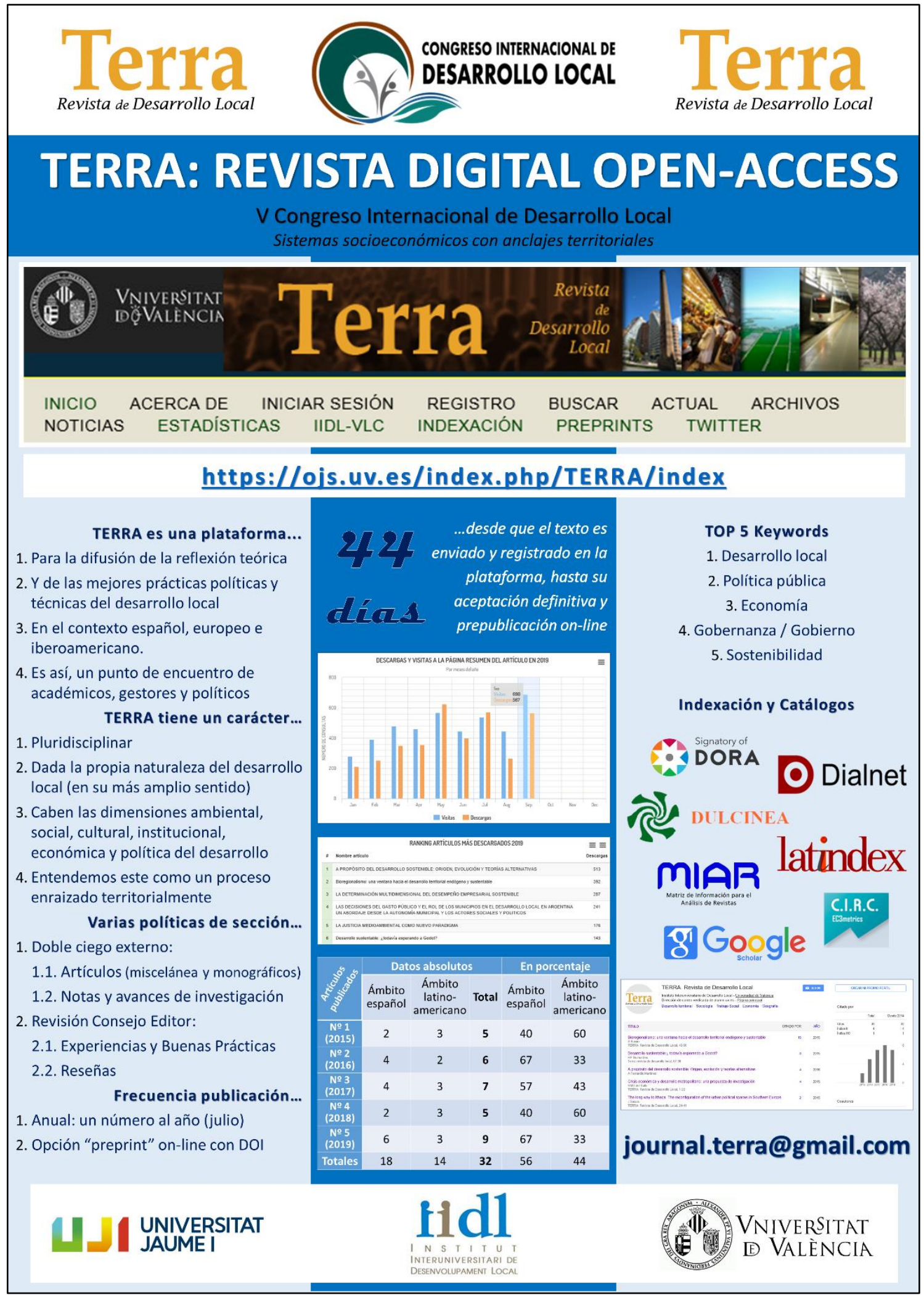

Fuente: elaboración propia. 
b) Conseguir la incorporación de TERRA al Índice de Referencia Europeo para las Humanidades y las Ciencias Sociales (ErihPlus), formar parte de las Bases de Datos y Directorios de Publicación Periódicas EBSCO, ProQuest, ELSERVIER y DOAJ, y lograr entrar en el Catálogo de Latindex (actualmente TERRA está recogida tan solo en el Directorio de dicha plataforma).

c) Abrir un perfil propio en redes sociales como, por ejemplo, Twitter, independiente de los perfiles con la que cuenta en la actualidad el IIDL de la sede de la Universitat de València y de la Universitat Jaume I. Se trata de una acción que muchas otras revistas del ámbito de Ciencias Sociales han llevado a cabo con éxito, y que además les permiten adjuntar otros indicadores de citación, como por ejemplo ALTMETRICS ${ }^{2}$. En ella, entre otros aspectos, se podrían difundir con rapidez y facilidad las "ideas clave" recogidas en los artículos, comunicar la convocatoria de números especiales, conseguir contactos con investigadores interesados en publicar sus trabajos (como vía complementaria a las ya en desarrollo), etc.

d) Conseguir la participación de toda la oferta formativa del IIDL en la dinámica de TERRA, en particular, como fuente de potenciales autores y/o revisores, completando así la colaboración actualmente existente en este sentido, procedente de dicha institución u otras similares ${ }^{3}$. Por un lado, con dos másteres de reciente creación y ofertados, por el momento, como título propio de ADEIT: el Máster de Gestión de Políticas para el Desarrollo del Territorio (deLOCAL), y el Máster en Desarrollo, Turismo y Cultura Española. Y, por otro lado, con el Programa de Doctorado en Desarrollo Local y Cooperación Internacional, en el que aparte de la Universitat de València y la Universitat Jaime I, se encuentra la Universidad Politécnica de Valencia, lo que podría contribuir a diversificar la procedencia de los potenciales autores, en particular desde el ámbito de trabajo del Desarrollo Local.

e) Establecer mecanismos de cooperación con cátedras institucionales-universitarias con evidente impacto en el estudio del Desarrollo Local, y pertenecientes además a la Universitat de València, aspectos que facilitaría los contactos iniciales. En concreto, se han identificado las siguientes cátedras: i) Cátedra Gobierno Abierto, Participación y Open Data; ii) Cátedra Luis Amigó; iii) Cátedra Prospect Comunitat Valenciana 2030; iv) Cátedra UNESCO de Estudios sobre el Desarrollo; v) Cátedra de Cooperación y Desarrollo Sostenible; vi) Cátedra Cultura Territorial Valenciana; y vii) Cátedra Cooperativa Caixa Popular del Desarrollo Territorial y Economía Social Valencianos.

\section{RESULTADOS}

Los resultados obtenidos de todas las acciones descritas en el apartado anterior sobre realizaciones, se sintetizan bien con los datos relativos al número de textos recibidos (sin distinguir si se trata de originales que terminan como artículos, notas, buenas prácticas o

\footnotetext{
${ }^{2}$ El comentario o discusión de un artículo puede ser visto como una métrica que muestra el impacto potencial del texto en cuestión. Las fuentes típicas de los datos para el cálculo de este indicador incluyen Facebook, Google+, Twitter, blogs científicos y páginas de Wikipedia.

${ }^{3}$ Esta se encuentra conformada por el Máster Oficial e Interuniversitario en Cooperación al Desarrollo, del Instituto Interuniversitario de Desarrollo Local de la Universidad de Valencia; el Máster Oficial en Planificación Territorial y Gestión Ambiental, del Centro Universitario Internacional de Barcelona (UNIBA); y el Máster en Desarrollo Local e Innovación Territorial, de la Facultad de Filosofía y Letras de la Universidad de Alicante.
} 
reseñas): 41 en total. Si de esta cifra eliminamos los cinco textos que están en proceso de evaluación en este mes de julio, y que de ser aceptados se publicarán en el siguiente número de la revista, el séptimo (diciembre de 2020), obtenemos unos resultados que podemos considerar de satisfactorios para una publicación todavía por indexar en las principales plataformas de referencia (Tabla 3).

Tabla 3. Datos relativos a los originales recibidos para la publicación del número 6

\begin{tabular}{|c|c|c|}
\hline Recibidos del 01 de julio de 2019 al 01 de julio de $2020(*)$ & 36 & $100 \%$ \\
\hline Aceptados para su publicación & 25 & $69,4 \%$ \\
\hline Rechazados & 11 & $30,6 \%$ \\
\hline
\end{tabular}

Fuente: elaboración propia a partir de los datos de gestión de TERRA. (*) La cifra real de textos recibidos es de 41, pero cinco de ellos se dirigen al número 7 de la revista debido a los procesos de evaluación.

De los 25 originales recibido y aceptados, 11 fueron propuestos inicialmente como artículos, si bien tras el proceso de evaluación externo, dos de ellos se dirigieron a la sección de Notas y Avances de Investigación, quedando los 9 restantes como Artículos; 4 de ellos se presentaron directamente a la sección de Experiencias y Buenas Prácticas, y 10 de ellos entraron como Reseñas, 9 de ellos de libros de reciente publicados (de 2018 en adelante), y una como síntesis de una jornada sobre desarrollo rural realizada en noviembre de 2019 en Algimia de Alfara (Valencia) (Tabla 4). Estas 10 reseñas inauguran por primera vez la sección de la revista de idéntico nombre que, si bien desde el principio activa, no tenía hasta ahora aporte alguno realizado.

Tabla 4. Publicaciones en TERRA por sección y año de publicación

\begin{tabular}{|c|c|c|c|c|c|c|c|c|c|}
\hline & \multicolumn{4}{|c}{ Datos absolutos } & \multicolumn{4}{c|}{ En porcentaje } \\
\hline & Artículos & Notas & BBPP & Reseñas & Total & Artículos & Notas & BBPP & Reseñas \\
\hline Número 1 (2015) & 4 & 0 & 1 & 0 & $\mathbf{5}$ & 80 & 0 & 20 & 0 \\
\hline Número 2 (2016) & 5 & 0 & 1 & 0 & $\mathbf{6}$ & 83 & 0 & 17 & 0 \\
\hline Número 3 (2017) & 7 & 0 & 0 & 0 & $\mathbf{7}$ & 100 & 0 & 0 & 0 \\
\hline Número 4 (2018) & 4 & 0 & 1 & 0 & $\mathbf{5}$ & 80 & 0 & 20 & 0 \\
\hline Número 5 (2019) & 6 & 1 & 2 & 0 & $\mathbf{9}$ & 67 & 11 & 22 & 0 \\
\hline Número 6 (2020) & 9 & 2 & 4 & 10 & $\mathbf{2 5}$ & 36 & 8 & 16 & 40 \\
\hline Totales & $\mathbf{3 5}$ & $\mathbf{3}$ & $\mathbf{9}$ & $\mathbf{1 0}$ & $\mathbf{5 7}$ & $\mathbf{6 2}$ & $\mathbf{5}$ & $\mathbf{1 6}$ & $\mathbf{1 7}$ \\
\hline
\end{tabular}

Fuente: elaboración propia a partir de: https://ojs.uv.es/index.php/TERRA/issue/archive.

En general, el tiempo de revisión y aceptación empleado para la publicación de estas 25 aportaciones se cifra en un total de 33 días, esto es, 10 días por debajo de los registros relativos al número anterior. Sin duda, consideramos que este resultado sobre la gestión de los textos recibidos, desde su recepción, búsqueda de revisores anónimos (habitualmente dos, si bien en algunos casos, también tres), evaluación por parte de los mismos de toda la documentación existente, respuesta a los autores con las sugerencias a realizar emitidas por los evaluadores (en caso de ser aceptados), y entrega de los textos reelaborados por parte de los autores, constituye una de las principales fortalezas de 
nuestra revista. De hecho, consideramos que se trata de uno de los aspectos que más debemos destacar, junto con la opción de pre-publicación con DOI antes apuntada, para poder conseguir incrementar la llegada de originales a TERRA o, cuanto menos, incorporar la misma al abanico de opciones valoradas para el envío de originales por parte de los investigadores (juniors y seniors) en Desarrollo Local.

Figura 4. Tiempo empleado en la publicación de originales en TERRA (días) según número/año

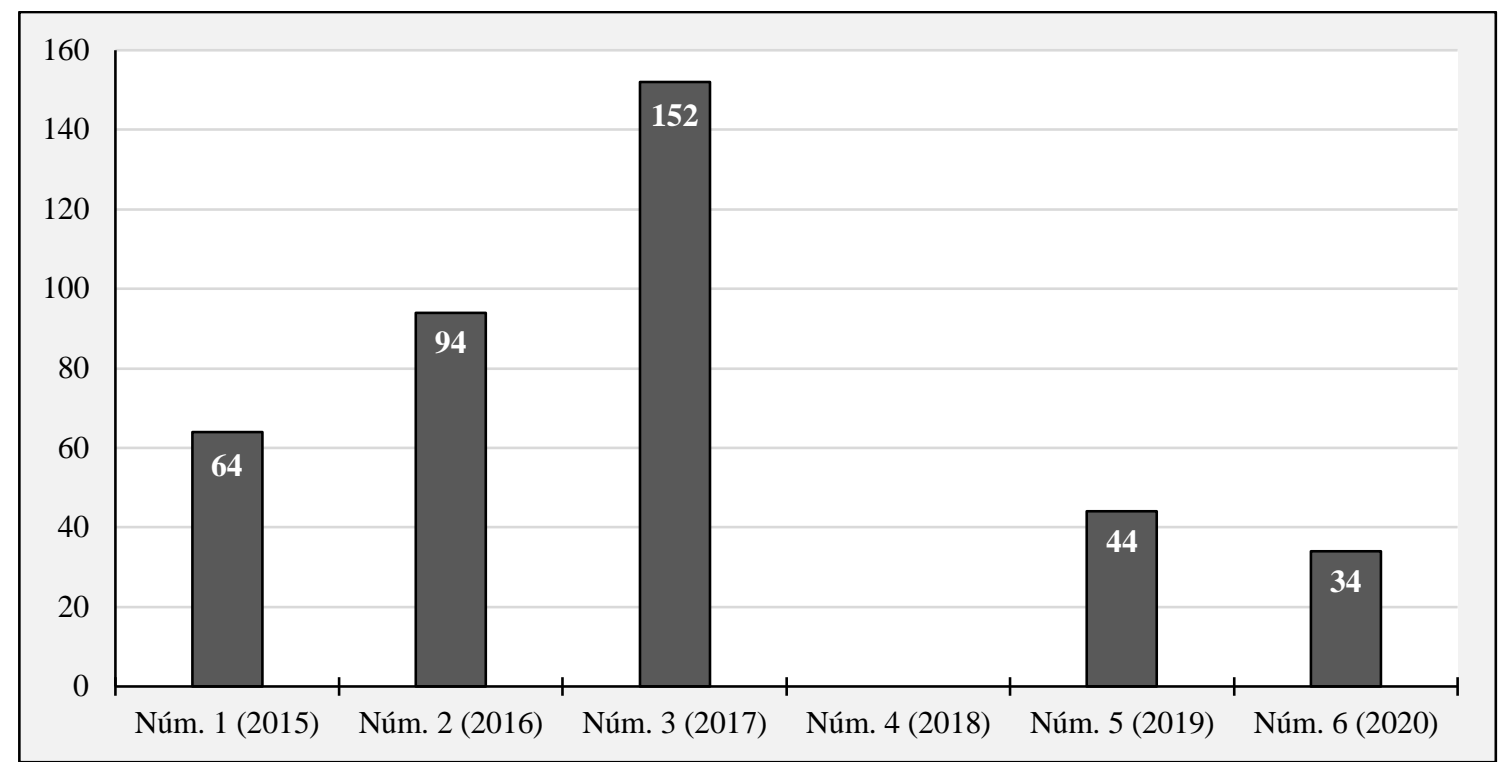

Fuente: elaboración propia a partir de: https://ojs.uv.es/index.php/TERRA/issue/archive. No se dispone de registros para el número 4 (2018).

Aparte de lograr el cierre del número actual con la cantidad de aportaciones más elevada y diversificada hasta la fecha (al contar con originales en todas sus secciones), otro de los resultados que evidencian un impacto relativamente positivo de las acciones desarrolladas se relaciona con el número de visitas a la web de la revista registradas desde el 01 de julio de 2019 al 30 de junio de 2020, y con la cantidad de textos descargados en idéntico período (Tabla 5).

Tabla 5. Consultas a la web de TERRA (visitas y descargas) de 2018 a 2020

\begin{tabular}{|l|c|c|c|c|}
\hline & $\begin{array}{c}\text { Visitas } \\
\text { (absolutas) }\end{array}$ & $\begin{array}{c}\text { Descargas } \\
\text { (absolutas) }\end{array}$ & $\begin{array}{c}\text { Visitas al } \\
\text { mes }\end{array}$ & $\begin{array}{c}\text { Descargas } \\
\text { al mes }\end{array}$ \\
\hline 01 enero 2018 a 31 de enero de 2019 (13 meses) & 3.967 & 2.487 & 305 & 191 \\
\hline 01 de febrero a 30 de junio de 2019 (5 meses) & 2.349 & 1.986 & 470 & 397 \\
\hline 01 de julio 2019 a 30 de junio de 2020 (12 meses) & 7.629 & 6.328 & 636 & 527 \\
\hline
\end{tabular}

Fuente: elaboración propia a partir de: https://ojs.uv.es/index.php/TERRA/statistics.

Este incremento señalado tanto en el número de originales recibidos y publicados, en todas las secciones, como de visitas y de descargas registradas, concuerda igualmente con el aumento experimentado de las citas que han recibido los textos publicados en los números anteriores de la revista (Tabla 6). 
Tabla 6. Métricas de TERRA en Google Académico

\begin{tabular}{|l|c|c|}
\hline & A 23 de julio de 2019 & A 23 de julio de 2020 \\
\hline Citas recibidas & 26 & 59 \\
\hline Índice H & 3 & 4 \\
\hline Índice i10 & 1 & 2 \\
\hline Número máximo de citas recibidas por un texto (*) & 13 & 18 \\
\hline Número de textos citados & 4 & 12 \\
\hline
\end{tabular}

Fuente: elaboración propia a partir de: https://scholar.google.es/citations?user=T3zUOrIAAAAJ\&hl=es. Nota (*): se trata del artículo titulado Bioregionalismo: una ventana hacia el desarrollo territorial endógeno y sustentable (Boisier, 2015).

Desde el punto de vista de la internacionalización de las participaciones, TERRA presente todavía cierto margen de mejora, puesto que la presencia de autores extranjeros (vinculados a centros de investigación ubicados fuera de España) aún es relativamente escasa (un $35 \%$ de las ocasiones) (Tabla 7). En cuanto a los contenidos la situación está más equilibrada, puesto que las temáticas y/o ejemplos empíricos externos al ámbito español y valenciano suponen casi la mitad de los textos publicados hasta el momento (Tabla 8). No obstante, el idioma de todos ellos es, salvo un aporte en el número 6 en catalán, el español, pese a que en la revista se puedan publicar textos en inglés, francés y portugués.

Tabla 7. Autores por centro de procedencia y número / año de publicación

\begin{tabular}{|c|c|c|c|c|c|}
\hline & \multicolumn{3}{|c|}{ Datos absolutos } & \multicolumn{2}{c|}{ En porcentaje } \\
\hline & Nacionales & Extranjero & Total & Nacional & Extranjero \\
\hline Número 1 (2015) & 3 & 3 & $\mathbf{6}$ & 50 & 50 \\
\hline Número 2 (2016) & 4 & 4 & $\mathbf{8}$ & 50 & 50 \\
\hline Número 3 (2017) & 7 & 7 & $\mathbf{1 4}$ & 50 & 50 \\
\hline Número 4 (2018) & 7 & 10 & $\mathbf{1 7}$ & 41 & 59 \\
\hline Número 5 (2019) & 11 & 5 & $\mathbf{1 6}$ & 69 & 31 \\
\hline Número 6 (2020) & 32 & 5 & $\mathbf{3 7}$ & 86 & 14 \\
\hline Totales & 64 & 34 & $\mathbf{9 8}$ & 65 & 35 \\
\hline
\end{tabular}

Fuente: elaboración propia a partir de: https://ojs.uv.es/index.php/TERRA/issue/archive

En cuanto a los contenidos publicados en las distintas secciones de TERRA, el análisis de las palabras clave de estos nos permite observar gran diversidad temática a partir de las cuales los distintos autores que han publicado en TERRA se aproximan al estudio del Desarrollo Local. De hecho, de las 284 palabras clave recogidas en los 57 textos publicados en estos seis números, el concepto de "Desarrollo Local" es sin duda el más repetido (14 ocasiones), seguido a cierta distancia de "Turismo" y "Gobernanza" (en 7 ocasiones), "Sostenibilidad" (6 ocasiones), "Política pública" (5 ocasiones), y "Participación" y "Gobierno" (4 ocasiones cada una) (Tabla 9). Sin duda, estos resultados demuestran la interdisciplinariedad del Desarrollo Local, y desde la que TERRA pretende aproximarse a su análisis, aprendizaje y difusión. 
Tabla 8. Publicaciones en TERRA por temática abordada y año de publicación

\begin{tabular}{|c|c|c|c|c|c|c|c|}
\hline & \multicolumn{4}{|c|}{ Datos absolutos } & \multicolumn{3}{c|}{ En porcentaje } \\
\hline & Nacional & Internacional & Genérico & Total & Nacional & Internacional & Genérico \\
\hline Número 1 (2015) & 1 & 0 & 4 & $\mathbf{5}$ & 20 & 0 & 80 \\
\hline Número 2 (2016) & 2 & 2 & 2 & $\mathbf{6}$ & 33 & 33 & 33 \\
\hline Número 3 (2017) & 2 & 3 & 2 & $\mathbf{7}$ & 29 & 43 & 29 \\
\hline Número 4 (2018) & 2 & 2 & 1 & $\mathbf{5}$ & 40 & 40 & 20 \\
\hline Número 5 (2019) & 6 & 2 & 1 & $\mathbf{9}$ & 67 & 22 & 11 \\
\hline Número 6 (2020) & 16 & 7 & 2 & $\mathbf{2 5}$ & 64 & 28 & 8 \\
\hline Totales & $\mathbf{2 9}$ & $\mathbf{1 6}$ & $\mathbf{1 2}$ & $\mathbf{5 7}$ & $\mathbf{5 1}$ & $\mathbf{2 8}$ & $\mathbf{2 1}$ \\
\hline
\end{tabular}

Fuente: elaboración propia a partir de: https://ojs.uv.es/index.php/TERRA/issue/archive

Tabla 9. Palabras clave empleadas en los textos aparecidos en TERRA (2015-2020)

\begin{tabular}{|c|c|c|c|c|}
\hline Orden & Palabras clave & Frecuencia & $\%$ & \% acumulado \\
\hline 1 & Desarrollo local & 14 & 4,9 & 4,9 \\
\hline 2 & Turismo & 7 & 2,5 & 7,4 \\
\hline 3 & Gobernanza & 7 & 2,5 & 9,9 \\
\hline 4 & Sostenibilidad & 6 & 2,1 & 12,0 \\
\hline 5 & Política pública & 5 & 1,8 & 13,7 \\
\hline 6 & Participación & 4 & 1,4 & 15,1 \\
\hline 7 & Gobierno & 4 & 1,4 & 16,5 \\
\hline 8 & Planificación & 3 & 1,1 & 17,6 \\
\hline 9 & Paisaje & 3 & 1,1 & 18,7 \\
\hline 10 & Movilidad & 3 & 1,1 & 19,7 \\
\hline 11 & Espacios rurales & 3 & 1,1 & 20,8 \\
\hline 12 & Economía & 3 & 1,1 & 21,8 \\
\hline 13 & Descentralización & 3 & 1,1 & 22,9 \\
\hline $14-219$ & Resto & 219 & 77,1 & 100,0 \\
\hline & & 284 & 100,0 & \\
\hline & & & &
\end{tabular}

Fuente: elaboración propia a partir de: https://ojs.uv.es/index.php/TERRA/issue/archive

Por último, recogemos a continuación el listado de evaluadores externos que han actuado desde el 01 de julio de 2018 hasta el 30 de junio de 2020, y que han contribuido a que TERRA se presente como una revista que cumple con todos los niveles de exigencia propios de las publicaciones basadas en la selección de originales para su publicación, a partir de un proceso de evaluación por pares ciegos. Desde aquí les agradecemos encarecidamente su inestimable colaboración a nuestro crecimiento, realizando una labor muchas veces desapercibida y, todavía, poco reconocida y valorada curricularmente.

1. Adrián Ferrandis Martínez. Universitat de València

2. Albert Moncusí Ferré. Universitat de València 
3. Alistair Adam Hernández. Universidad de Ciencias Aplicadas y Arte (HAWK) (Alemania)

4. Álvaro Sánchez Crispín. Universidad Nacional Autónoma de México

5. Antonio Martínez Puche. Universidad de Alicante

6. Claro Águila Pérez. Universidad Agraria de la Habana (Cuba)

7. David Vladimir Cartuche Paqui. Universidad Nacional de Loja (Ecuador)

8. Efraín García Palacios. Universidad de Córdoba

9. Emilia Guisado Pintado. Universidad de Sevilla

10. Esther Rando Burgos. Universidad de Málaga

11. Francisco Almarcha Martínez. Universidad de Alicante

12. Francisco Navarro Valverde. Universidad de Granada

13. Gabriela García Vélez. Universidad de Cuenca (Ecuador)

14. Ghaleb Fansa Saleh. Universitat de València

15. Ibán Vázquez González. Universidad de Santiago de Compostela

16. Jesús Burgueño Rivero. Universitat de Lleida

17. Joan Carles Membrado Tena. Universitat de València

18. Joan del Alcazar. Universitat de València

19. Jorge Luis Sosa. Universidad Autónoma de Ciudad Juárez (México)

20. José Antonio Nieto Calmaestra. Delegación Territorial de Fomento Vivienda y Ordenación del Territorio, Granada.

21. José Javier Serrano Lara. Universitat de València

22. José Luís García Cuesta. Universidad de Valladolid

23. José Luís Sánchez Hernández. Universidad de Salamanca

24. José María Gómez Espín. Universidad de Murcia

25. José Vicente Pérez Cosín. Universitat de València

26. José Vicente Sánchez Cabrera. Universitat de València

27. Juan Ignacio Plaza Gutiérrez. Universidad de Salamanca

28. Julio Fernández Portela. Universidad Nacional de Educación a Distancia (UNED)

29. Karla Violeta Pillado Albarrán. Universidad Autónoma del Estado de México

30. Libys Zúñiga Igarza. Universidad de Holguín (Cuba)

31. Louis Valentín Mballa. Universidad Autónoma de San Luis Potosí (México)

32. Luís Miguel Sánchez Escolano. Universidad de Granada

33. María Cleofe Villarín Claveríaz. Universidad de Sevilla

34. María de la O Barroso González. Universidad Internacional de Andalucía

35. María Eugenia Campoy Gómez. Instituto Tecnológico de Oaxaca (México)

36. María Hernández Hernández. Universidad de Alicante 
37. María Sanagustín Fons. Universidad de Zaragoza

38. María Teresa Riquelme Quiñonero. Universidad de Alicante

39. Marta Pallarès-Blanch. Universitat Autònoma de Barcelona

40. Mercedes Botija Yagüe. Universitat de València

41. Michel Bregolin. Universidad Caxias Do Sul (Brasil)

42. Oriol Estela Barnet. Cátedra Barcelona-UPF de Política Económica Local

43. Pablo Giménez Font. Universidad de Alicante

44. Rodrigo Arocena. Universidad de la República del Uruguay (Uruguay)

45. Rosario Asián Chaves. Universidad de Sevilla

46. Santiago Cantarero Sanz. Universitat de València

47. Sara Martínez Pellegrini. El Colegio de la Frontera Norte (México)

48. Venancio Cuevas-Reyes. Instituto Nacional de Investigaciones Forestales, Agrícolas y Pecuarias (México)

49. Vicent Querol Vicente. Universitat Jaume I de Castelló

50. Vicente Alanoca Arocutipa. Universidad Nacional del Altiplano (Puno-Perú)

51. Xavier Amat Montesinos. Universidad de Alicante

52. Xavier Negrillo-Deza. Universidad Politécnica De Madrid

Valencia, 27 de julio de 2020 